\title{
A Fuzzy Petri-Net Based Conceptual Model for Risk Prediction in Enterprise Resource Planning Acquisition Decisions for Small and Medium Enterprises
}

\author{
Vijayakumar Bharathi, Dhanya Pramod and Ramakrishnan Raman \\ Symbiosis Centre for Information Technology, \\ Faculty of Computer Studies, Symbiosis International University, Pune, India
}

Received 2013-03-11, Revised 2013-03-17; Accepted 2013-03-18

\begin{abstract}
This study identifies and evaluates certain critical pitfalls in the acquisition phase of ERP adoption in Small and Medium Enterprises (SMEs) and develops a model for evaluating the risk of failure. On the acquisition stage a set of risks has been identified based on past research work and each of these risks have been explained through a set of well-defined risk factors or pitfalls. An evaluation model based on the concept of Fuzzy Petri Nets (FPN) is proposed in this study. FPN is used because it is simple and efficient and can quantitatively evaluate the risks inherent in the acquisition stage of ERP adoption by SMEs.
\end{abstract}

Keywords: ERP Adoption, SMEs, Failure Prediction, Risk, Fuzzy Petri-Nets, Acquisition

\section{INTRODUCTION}

Integration of core business process is one of the critical factors that will enable organizations to optimize the utilization of $5 \mathrm{M}$ resources namely Men, Money, Machines, Materials and Methods. Business Process integration enables businesses to have transparency, clarity and speed in information flow across the processes, through the central creation and common sharing of data that gets accumulated in a database. The central creation and common sharing of data across the length, breadth and height of the organization is enabled through Enterprise Resource Planning System (ERP). ERP systems have gain a high prominence through enabling companies to integrate business processes, streamline their operations and also leverage the value of seamless integration. It is critical to understand the need and alignment of the ERP system with the business needs. Hence ERP acquisition decision should keep in mind both the available software functionalities inbuilt in the ERP system and also the expectation of the business. Some of the key factors in ERP selection were identified as vendor's reputation, internationality, sales references and successfully completed projects in the same industry (Karsak and Ozogul, 2009). The business environment has been dramatically transformed due to the severe market competition which has forced the companies to look into perspectives of growth like total costs reduction, maximize return on investment, shorten lead times and be more responsive to customer demands. The use of an effective ERP system had been accepted to be an enhancing factor for competitive advantage in highly dynamic markets (Wei et al., 2005). It has been found that SMEs operating in India and the ERP vendors often fail in recognizing, the technical, financial and organizational impacts during ERP adoption which results overall failure of the expectation with ERP realization. The study called for an in-depth evaluation of the ERP software and vendor capabilities so as to reduce the gap between ERP functionalities and business requirements. Some of the critical factors for ERP selection included adequate and thorough knowledge ERP products, budget planning and appropriate training (Ganesh and Mehta, 2010).

Corresponding Author: Vijayakumar Bharathi, Symbiosis Centre for Information Technology, Faculty of Computer Studies, Symbiosis International University, Pune India 
This research paper aims at identifying and evaluating certain critical factors that can impact the ERP acquisition decisions by SMEs. The factors are selected based on the extensive review of literature. An evaluation and measurement model has been conceptually developed using Fuzzy Petri Nets the explanation of which has been given in the subsequent sections.

\subsection{Literature Review}

Considerable research has been done to foresee and analyze ERP risks in the acquisition of the right or appropriate ERP for SMEs. Realistic estimate of process value addition, usage of lean tools for value stream mapping and process flow diagrams have been suggested as key factors for ERP selection for SMEs. The key performance indicators out of acquiring an ERP should be carefully studied from various perspectives like the uniqueness of business, scale of operations, organization culture (Aman et al., 2007).

It is a complex and costly process for SMEs to implement ERP systems. Several lessons of the past indicate morals of serious failures. Identification of Critical Failure Factors (CFFs) is equally significant as Critical Success Factors (CSFs). The study found that there still major consideration to be addressed in developing countries while implementing ERP Systems unlike the technologically leading countries. Amongst the 47 CFFs tested in Iranian SMEs two factors namely conflicts between organization and vendors and poor vendor selection has been attributed to ERP Acquisition phase (Amin et al., 2012). Amongst the six stages of ERP implementation namely, initiation, adoption, adaptation, acceptance, routinization and infusion, vendor support and use of vendor tools are specifically found to be important during the first three stages of implementation and interestingly less in the later stages. The importance of consultants was found to be very high in the infusion stage of ERP implementation (Somers and Nelson, 2004). ERP is recognized as a strategic IT initiative having long-term irreversible implications. It is very critical to understand the underlying risks that can surface at each and every stage of ERP Adoption life cycle of the SMEs. Though various tools have been used for risk assessment, comparatively less number of researchers has used Petri-net approaches, in particular Fuzzy Petri-Net (FPN) approach for risk prediction. A Fuzzy Petri-Net based model was proposed to evaluate and measure the risk on the risks associated with the planning phase of ERP Adoption in SMEs (Vijayakumar et al., 2012a). The fuzzy theories of ERP critical success factors reduce ambiguities and uncertainties which are inherent in qualitative data analysis. A research study on critical success factors in a refrigerator company in Iran used a Fuzzy Analytical Hierarchy Process (FAHP) approach to evaluate and prioritize ERP Critical Success Factors (CSFs) (Amalnick et al., 2010). In order to assess the current state of organizational readiness to implement an ERP system a Fuzzy Analytic Network Process based framework was developed. The framework decomposed ERP readiness into three parts namely "project management readiness", "organizational readiness" and "change management readiness". There were five main categories of CSFs namely project, vision and goals, systems and processes, culture and structures and human resources which were selected to study the assessment parts mentioned in the framework (Razmi et al., 2009). Since ERP implementation decision can have strategic and irrevocable implications to organizations it is important to study carefully the associated uncertainties in orders to capture the upside benefits and to control the downside losses. Comparatively a lot of research literatures are available more for large companies than for SMEs. Considering the limited expertise on financial and human capital a useful implementation guidelines and decision support model was found essential for the SMEs while ERP adoption. A Fuzzy based Real Option Theory was developed for facilitating decision support to the SME management during the various stages of ERP implementation (You et al., 2012). A Unified Theory on CSFs for ERP adoption in SMEs was established with five decision areas of ERP namely Planning, Acquisition, Implementation, Usage and Percolation and Extension within which a set of 39 critical success factors were identified. The decision drivers in each of the five phases were impacted by a set of identifying critical success factors (Vijayakumar and Shrikant, 2009). 30 CSFs were identified from the research literature and were grouped under five sequential decision stages of ERP Adoption namely Planning, Acquisition, Implementation, Usage and Percolation and Extension. Using AHP these CSFs were ranked based on the popularity of these CSFs in the existing literature and their practical validity was tested by comparing the conceptual ranking and prioritizing of the CSFs to a sample of SMEs (Vijayakumar et al., 2012b; Vijayakumar and Shrikant, 2012). An integrated decision support system to assist enterprise software selection with due consideration to both qualitative and quantitative objectives was developed. The system provided an objective hierarchy structure of the selection problem of ERP with regard to complete functional and non-functional suitability and reducing the total cost of ownership and implementation time (Sen et al., 2009).

\subsection{The Fuzzy Petri-Net Model for Risk Prediction in Erp Adoption for Smes}

The proposed fuzzy Petri Net model contains the following definitions.

\subsection{Fuzzy Petri Net}

This represents fuzzy production rules of a rule-based system. It contains two types of node; places and transitions wherein circle represented place and the bar 
represents transitions. Each place may or may not contain token associated with a truth value between 0 and 1 . The transition has a certainty factor associated between 0 and 1 and directed arcs connect places to transition.

\subsection{Definition and Symbols}

A fuzzy Petri net structure can be defined as an 8-tuple:

$\mathrm{FPN}=(\mathrm{P}, \mathrm{T}, \mathrm{D}, \mathrm{I}, \mathrm{O}, \mathrm{f}, \alpha, \beta)$

Where:

$\mathrm{P}=\mathrm{p} 1, \mathrm{p} 2 \ldots \mathrm{pn}\} \quad=$ A finite set of places

$\mathrm{T}=\{\mathrm{t} 1, \mathrm{t} 2, \ldots \mathrm{tm}\} \quad=$ A finite set of transitions

$\mathrm{D}=\{\mathrm{d} 1, \mathrm{~d} 2, \ldots \mathrm{dn}\}=$ A finite set of propositions

$\mathrm{P} \cap \mathrm{T} \cap \mathrm{D}=\Phi,|\mathrm{P}|=|\mathrm{D}|$

$\mathrm{I}: \mathrm{T} \rightarrow \mathrm{P} \infty \quad=$ The input function, a mapping from transitions to bags of places

$\mathrm{O}: \mathrm{T} \rightarrow \mathrm{P} \infty \quad$ = The output function, a mapping from transitions to bags of places

$\mathrm{F}: \mathrm{T} \rightarrow[0,1] \quad=\mathrm{An}$ association function, a mapping from transitions to real values between 0 and 1

$\alpha: \mathrm{P} \rightarrow[0,1] \quad=$ An association function, a mapping from places to real values between 0 and 1

$\beta: \mathrm{P} \rightarrow \mathrm{D} \quad=\mathrm{An}$ association function, an objective mapping from places to propositions

\subsection{Fuzzy Production Rule}

It explains the fuzzy relationship between two propositions.

If $d_{j 1}$ or $d_{j 2} \ldots . d_{j n}$ then $d_{k}\left(C . F=\mu_{I}\right)$. This rule type is modeled in fuzzy petri-net Fig. 1A and the fuzzy reasoning process of this type of rule is modeled in Fig. 1B.

If $d_{j 1}$ and $d_{j 2} \ldots . . d_{j n}$ then $d_{k}\left(C . F=\mu_{I}\right)$. This rule type is modeled in fuzzy Petri Net Fig. 2A and the fuzzy reasoning process of this type of rule is modeled in Fig. 2B.

From past research work five distinct phases of ERP Adoption have been identified namely planning, acquisition, implementation, usage and extension. Amongst these the second phase of ERP Adoption namely the Acquisition phase is considered here for modeling. A set of five risks has been identified in this phase. Certain attributes have been defined for each of the five risks in the acquisition phase of ERP adoption.

Many of the SMEs are still in the primitive stages of ERP adoption. The SMEs are skeptical regarding certain key factors regarding ERP selection. They are less aware on the choice of the right ERP product, the right vendor, the right consultant, a reconciliation of their existing IT set-up with what is expected for implementing ERP. The information flow in a majority of SMEs is dependent on manual and spreadsheets based communication processes and hence ERP is not known widely in the SMEs as a strategic IT enabler. This lack of adequate technology infrastructure is what we define as the first risk that hinders the acquisition of ERP for the SMEs. Interestingly, this risk comes with a variety of factors which contributes from both internal and external perspectives to the organization. Some of the major factors apart from lack of adequate technology infrastructure are inadequate selection methods for ERP product and delivery, inadequate knowledge of ERP vendor selection, ineffective external consultant and inadequate support of SME industry associations.

The Table 1 given below explains the risk and its attributes.

\subsection{ERP Failure Prediction Model}

The fuzzy Petri Net based model is explained below.

At place $\mathrm{A}_{0} \quad \mathrm{~d}_{0}$ is the risk of failure in the Acquisition Phase of ERP Adoption.

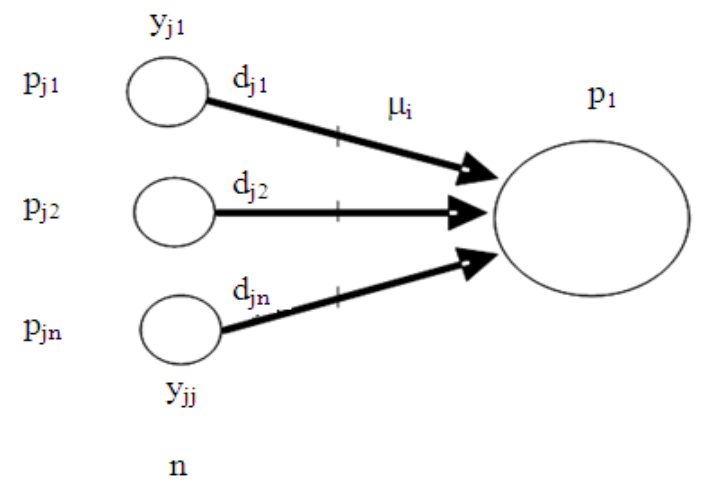

Fig. 1A. Fuzzy Rule Type

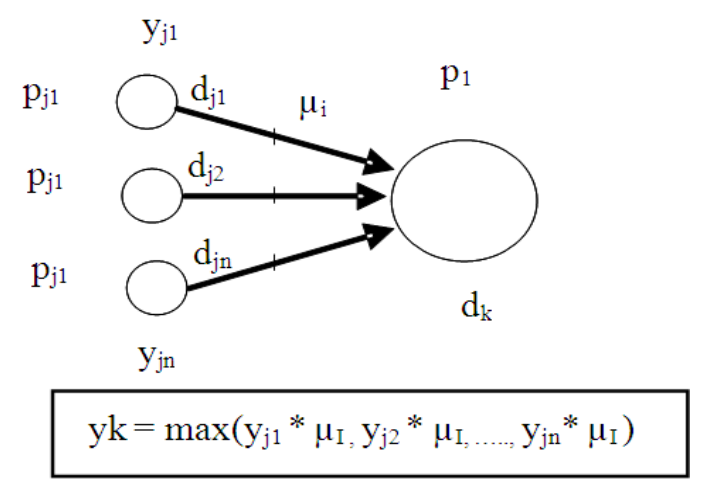

Fig. 1B. Fuzzy Reasoning Process 
Table 1. Risk and risk factors

\begin{tabular}{|c|c|c|c|c|}
\hline S. No & Risk & Symbol & Risk factors & Symbol \\
\hline 1 & $\begin{array}{l}\text { Lack of adequate } \\
\text { technology infrastructure }\end{array}$ & $\mathrm{A}_{1}$ & $\begin{array}{l}\text { Outdated, disparate state of current } \\
\text { IT systems Transaction processing } \\
\text { and consolidation manually }\end{array}$ & $\begin{array}{l}\mathrm{a}_{11} \\
\mathrm{a}_{12}\end{array}$ \\
\hline 2 & $\begin{array}{l}\text { Inadequate knowledge on ERP } \\
\text { product and delivery selection }\end{array}$ & $\mathrm{A}_{2}$ & $\begin{array}{l}\text { Lack of expertise on identifying, } \\
\text { scrutinizing \& selection of ERP. } \\
\text { No or little knowledge about SaaS/Cloud models } \\
\text { No study on ERP fitment to own organization }\end{array}$ & $\begin{array}{l}a_{21} \\
a_{22} \\
a_{23}\end{array}$ \\
\hline 3 & $\begin{array}{l}\text { Inadequate knowledge } \\
\text { on ERP vendor selection }\end{array}$ & $\mathrm{A}_{3}$ & $\begin{array}{l}\text { No knowledge on vendor capability analysis } \\
\text { No audit on success rates implementation and support } \\
\text { No due diligence study on vendor }\end{array}$ & $\begin{array}{l}a_{31} \\
a_{32} \\
a_{33}\end{array}$ \\
\hline 4 & Ineffective external consultant & $\mathrm{A}_{4}$ & $\begin{array}{l}\text { No or less interaction with process owners } \\
\text { Poor audit on the existing IT infrastructure } \\
\text { Less knowledge about SME operations }\end{array}$ & $\begin{array}{l}a_{41} \\
a_{42} \\
a_{43}\end{array}$ \\
\hline 5 & $\begin{array}{l}\text { Inadequate support from } \\
\text { SME Industry Associations }\end{array}$ & $\mathrm{A}_{5}$ & $\begin{array}{l}\text { No or very little experience on ERP consulting. } \\
\text { Lack of initiatives on ERP awareness and benefits No } \\
\text { mechanism to track and maintain ERP } \\
\text { success/failures of SME members }\end{array}$ & $\begin{array}{l}a_{51} \\
a_{52} \\
a_{53}\end{array}$ \\
\hline
\end{tabular}

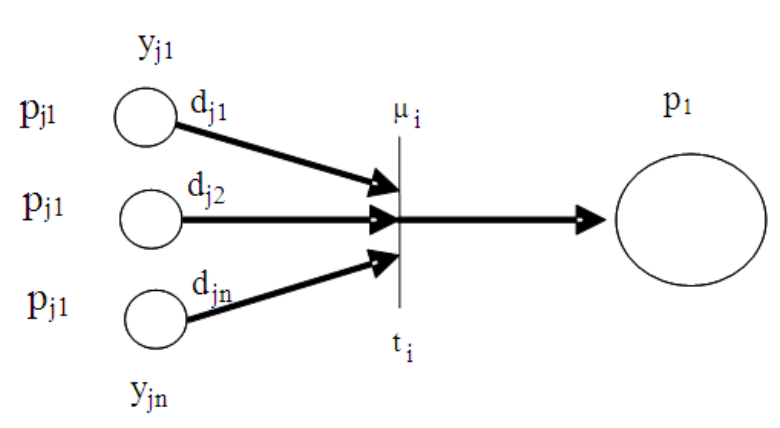

Fig. 2A. Fuzzy Rule Type

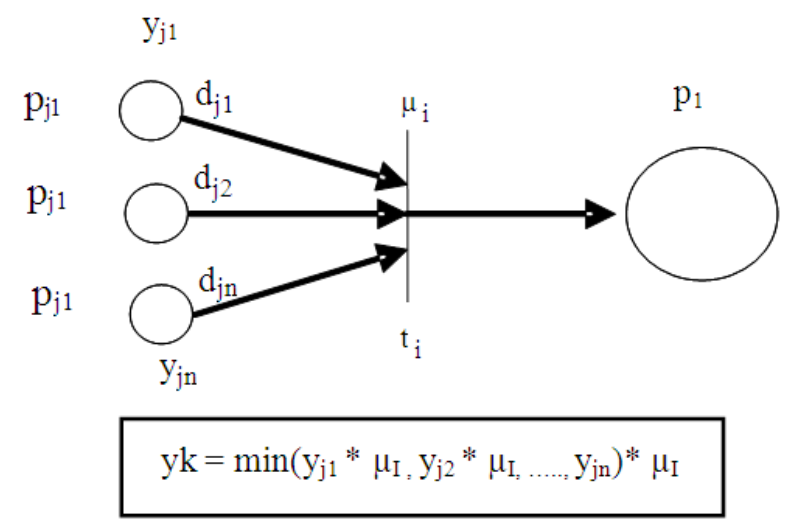

Fig. 2B. Fuzzy Reasoning Process

At place $A_{i}(i=1,2 \ldots ., 5) . D_{i}$ represents Risks identified that are associated with the Acquisition Phase. These factors will collectively lead to validate the risks of failure.
At place $a_{i j}(i=1,2 \ldots \ldots, 5),(j=1,2 \ldots \ldots n), d_{i j}$ represent the risk factor and $\mathrm{n}$ represents the number of risk factors identified to explain the risks.

At place $a_{i j k}(i=1,2 \ldots . .5),(j=1,2 \ldots . . n)(k=1$, 2.....m) where $d_{i j k}$ represents the responses (choices) for a risk factor viz., Strongly Agree (SA), Agree (A), Disagree (D) and Strongly Disagree (SD) and $\mathrm{m}$ represents the number of choices (here 4 choices) for recording the response to a risk factor. SA and A are defined as negative which implies that they will contribute/lead to risk while D and SD are defined as positive because they will not contribute/lead to risk in this study.

$\mathrm{D}_{\mathrm{ijk}}(\mathrm{i}=1,2 \ldots \ldots, 5),(\mathrm{j}=1,2 \ldots \ldots)(\mathrm{k}=1,2 \ldots \ldots \mathrm{m})$ where $d$ represents the percentage of responses (Ratio of number of responses to an option to the total responses recorded for all the options). If the percentage of responses are evenly spread in all the four options namely $\mathrm{SA}, \mathrm{A}, \mathrm{D}$ and $\mathrm{SD}$ or if the sum of the response proportion of negative and positive options is equal $(\mathrm{SA}+\mathrm{A})=$ $(\mathrm{D}+\mathrm{SD})$ then that factor may or may not contribute towards risk. If for a risk factor, the proportion of responses in $\mathrm{SA}$ and $\mathrm{A}$ is greater than $\mathrm{D}$ and $\mathrm{SD}$, then that factor will contribute towards risk and vice versa.

$\mu_{\mathrm{i}}(\mathrm{i}=1,2 \ldots . .9)$ represents the certainty factor, $\mu_{\mathrm{i}} \mathrm{C}$ $[0,1]$ that is the strength of the belief in the rule.

$\mathrm{T}_{1}(\mathrm{i}=1,2 \ldots . .9)$ represents the transition from input place to output place.

$\mu_{\mathrm{ij}}(\mathrm{i}=1,2 \ldots \ldots 9),(\mathrm{j}=1,2 \ldots . \mathrm{n})$ where $\mu$ represents the certainty factor of the responses to fire a risk factor. In this study, for illustration purpose 9 (as a proportion 0.9 ) is fixed throughout, because it is assumed that the respondents are well informed and are fully aware of the context and their responses can be highly dependable. The model for the Risk and the Associated Risk Factors is given below in Fig. 3 . 
In the acquisition phase of ERP adoption $\left(\mathrm{A}_{0}\right)$, the risk known as lack of adequate technology infrastructure $\left(A_{1}\right)$ is explained by means of two risk factors namely the outdated and disparate state of current IT systems $\left(\mathrm{d}_{11}\right)$ and transaction processing and consolidation manually $\left(d_{12}\right)$. The values for predicting the risk is based on the responses which range from strongly agree to strongly disagree $\left(d_{111} \ldots d_{114}\right)$ given by the respondents. For example in $d_{11}$ if $35 \%$ of the respondents say strongly agree then the value of $d_{111}$ is 0.35 and the balance $65 \%$ of respondents say agree then the value of $d_{112}$ is 0.65 . The strength of responses or truth value is denoted by $\mu$, the value of which is the weight assigned based on the dependability of the responses. For example if $\mu_{11}$ is assigned a value of 0.9 then it implies a high degree of dependability of responses. Then the value $\mathrm{y}_{11}$ is a maximum of $(0.35 * 0.9)$ and $(0.65 * 0.9)$ which is 0.585 . This value of 0.585 is arrived from the response option Agree (A). As said earlier, since SA and A denotes negative it implies that disparate state of current IT systems $\left(d_{11}\right)$ is one of the critical factors leading to risk of Lack of adequate technology infrastructure $\left(A_{1}\right)$.

Similarly the values of the other risk factor can be arrived based on the proportion of responses. Then the maximum of the proportion-of-response values multiplied by the $\mu_{12}$ value will determine whether that risk factor would contribute to the risk or otherwise.

Inadequate knowledge on ERP product and delivery selection $\left(\mathrm{A}_{2}\right)$ is explained through three risk factors namely lack of expertise in identifying, scrutinizing and selection of ERP $\left(\mathrm{d}_{21}\right)$, no or little knowledge about SaaS/Cloud models $\left(\mathrm{d}_{22}\right)$ and no study on ERP fitment to own organization $\left(d_{23}\right)$. Through the responses ranging from strongly agree to strongly disagree we can predict the value of risk factors. For instance, in $\mathrm{d}_{22} 20 \%$ of the respondents agree then the value of $\mathrm{d}_{221}$ is 0.20 and $50 \%$ of the respondents disagreeing then the value of $\mathrm{d}_{223} 0.50$ and $30 \%$ of the respondents strongly disagreeing then the value of $\mathrm{d}_{224}$ is 0.30 . If $\mu_{22}$ is assigned a value of 0.9 then value $\mathrm{y}_{22}$ is the greater of $(0.2 * 0.9),(0.5 * 0.9)$ and $(0.3 * 0.9)$ which is 0.45 . The value of 0.45 is maximum and it is arrived from option D. As defined earlier, D is positive which denotes that no or little knowledge about SaaS/Cloud models $\left(\mathrm{d}_{22}\right)$ will not lead to risk of Inadequate ERP product and delivery selection $\left(\mathrm{A}_{2}\right)$.

Similarly the value of other risk factors $d_{21}$ and $d_{23}$ can be arrived based on the proportion of responses. Then the maximum of the proportion-of-response values multiplied by $\mu_{21}, \mu_{23}$ values respectively will determine whether $d_{21}$ and $d_{23}$ will contribute or will also not contribute to the risk.
Inadequate knowledge on ERP vendor selection $\left(\mathrm{A}_{3}\right)$ is supported with three risk factors namely no knowledge on vendor capability analysis $\left(\mathrm{d}_{31}\right)$, no audit on the success rates of implementation and support $\left(\mathrm{d}_{32}\right)$, no due diligence study on the vendor $\left(\mathrm{d}_{33}\right)$. Based on respondents' perceptions ranging from strongly agree to strongly disagree we can arrive at the value of risk factors. Say for example for $\mathrm{d}_{31}$ if $10 \%$ of respondents strongly agree the value of $\mathrm{d}_{331}$ is $0.10,70 \%$ of them agree then value of $d_{332}$ is 0.70 and $20 \%$ of them strongly disagree the value of $d_{334}$ is 0.20 . The $\mu_{31}$ value is assigned as 0.9 , so the value of $\mathrm{y}_{31}$ will be the maximum of $(0.10 * 0.9),(0.70 * 0.9)$ and $(0.20 * 0.9)$ which is 0.63 .

The value of 0.63 is arrived out of the response option agree hence it can be understood that the risk factor no knowledge on vendor capability analysis $\left(\mathrm{d}_{31}\right)$ will contribute to the risk of Inadequate knowledge to the ERP vendor selection $\left(\mathrm{A}_{3}\right)$. Similarly, maximum value of $y_{32}$ and $y_{33}$ of other two risk factors $d_{32}$ and $d_{33}$ is calculated as a product of proportion-of-responses value and $\mu_{32}$ and $\mu_{33}$ respectively. Based on the positive and negative perspectives the y values will determine whether these risk factors will lead to or not lead to the risk.

Ineffective external consultant $\left(\mathrm{A}_{4}\right)$ is defined through three risk factors namely no or less interaction with process owners $\left(\mathrm{d}_{41}\right)$, poor audit of the existing IT infrastructure $\left(\mathrm{d}_{42}\right)$, less knowledge about SME operations $\left(\mathrm{d}_{43}\right)$. The values of the risk factors can be arrived from the responses ranging from strongly agree to strongly disagree. For $\mathrm{d}_{41}$, for example if the responses are evenly spread across all the options, that is $25 \%$ in each of the four options, then that factor will not be taken into consideration for determination of risk.

In the same way the value of other risk factor $\mathrm{d}_{42}$ can be calculated on the basis of response proportion. The value of $\mathrm{y}_{42}$ will be the maximum of proportion-of-responses and $\mu_{42}$ of 0.9 of that risk factor. The negative or positive attribute as defined earlier will determine whether the factor would contribute to the risk $\left(\mathrm{A}_{4}\right)$ or otherwise.

Inadequate support of SME Industry Associations $\left(A_{5}\right)$ is explained through no or very little experience on ERP consulting $\left(\mathrm{d}_{51}\right)$, lack of initiatives on ERP awareness and benefits $\left(\mathrm{d}_{52}\right)$ and no mechanism to track and maintain ERP success/failures of SME members $\left(\mathrm{d}_{53}\right)$. The risk-factors values can be arrived from the given range of responses from strongly agree to strongly disagree. For $\mathrm{d}_{51}$ for example if $20 \%$ of the respondents strongly agree the value of $d_{511}$ is 0.2 while $80 \%$ agree the value of $d_{512}$ is 0.8 . 


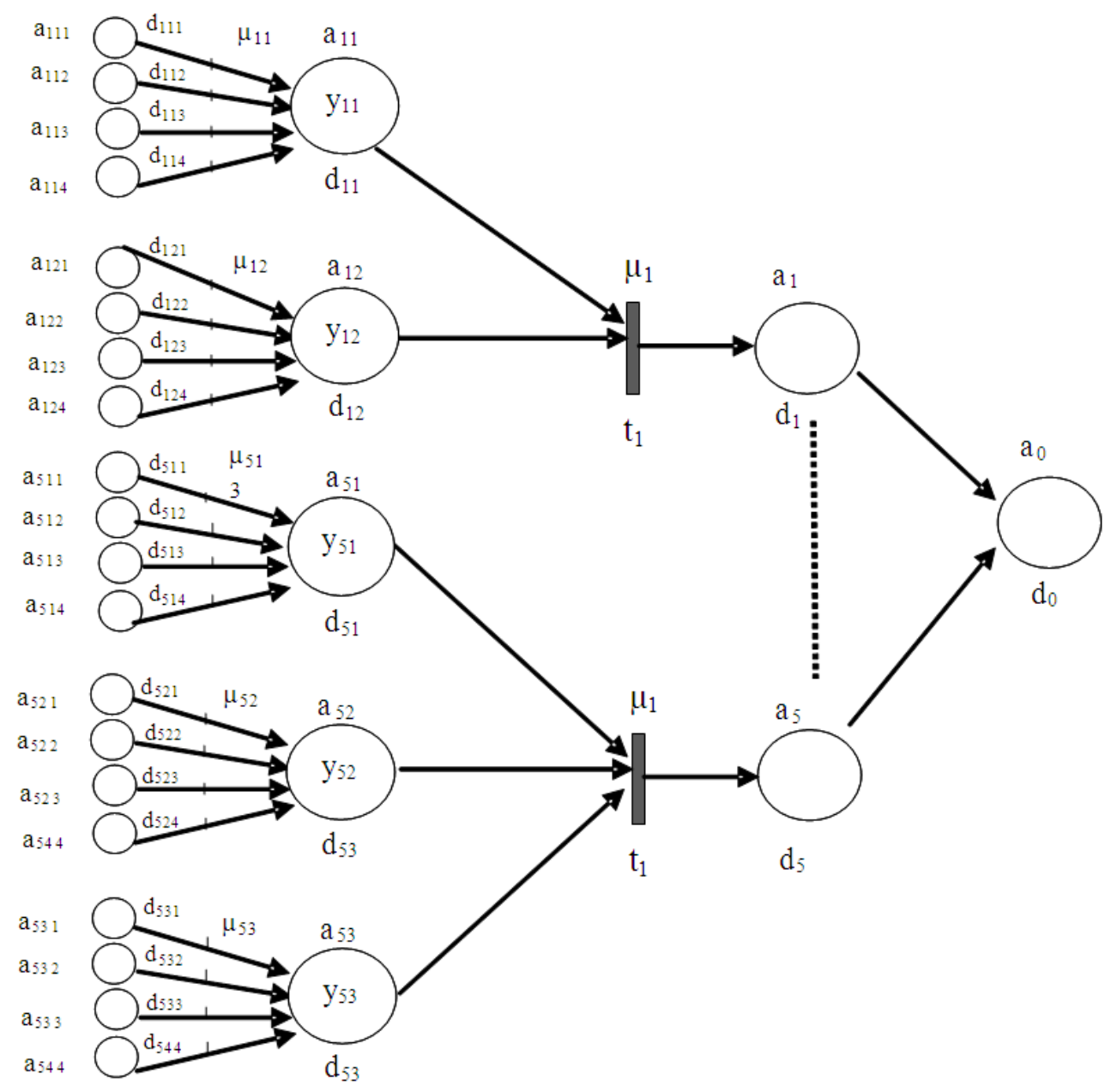

Fig. 3. Fuzzy petri-net model

Table 2. Values of risk factors

\begin{tabular}{|c|c|c|c|c|c|}
\hline S. No. & Risk symbol & Risk factor & --- & - & $+/-* *$ \\
\hline 1 & $\mathrm{~A}_{1}$ & $a_{11}$ & $\mathrm{y}_{11}$ & 0.585 & - \\
\hline 2 & $\mathrm{~A}_{2}$ & $\begin{array}{l}a_{12} \\
a_{21} \\
a_{22}\end{array}$ & $\begin{array}{l}\mathrm{y}_{12} 2 \\
\mathrm{y}_{21} \\
\mathrm{y}_{22}\end{array}$ & 0.45 & + \\
\hline 3 & $\mathrm{~A}_{3}$ & $\begin{array}{l}a_{23} \\
a_{31} \\
a_{32}\end{array}$ & $\begin{array}{l}\mathrm{y}_{23} \\
\mathrm{y}_{31} \\
\mathrm{y}_{32}\end{array}$ & 0.63 & - \\
\hline 4 & $\mathrm{~A}_{4}$ & $\begin{array}{l}a_{41} \\
a_{42}\end{array}$ & $\begin{array}{l}y_{33} \\
y_{41} \\
y_{42}\end{array}$ & NA* & NA* \\
\hline 5 & $\mathrm{~A}_{5}$ & $\begin{array}{l}a_{43} \\
a_{51} \\
a_{52} \\
a_{53}\end{array}$ & $\begin{array}{l}\mathrm{y}_{43} \\
\mathrm{y}_{51} \\
\mathrm{y}_{52} \\
\mathrm{y}_{53}\end{array}$ & $\begin{array}{l}0.72 \\
\text { NA* }\end{array}$ & NA* \\
\hline
\end{tabular}

The value of $\mu_{51}$ being 0.9 the value of $y_{51}$ will be the maximum of $(0.2 * 0.9)$ and $(0.8 * 0.9)$ which is 0.72 . In this outcome since 0.72 is arrived out of a negative attribute of responses namely agree, this risk factor no or very little experience on ERP consulting will contribute towards the risk $\left(\mathrm{A}_{5}\right)$.

In the same way the value of other risk factors $d_{52}$ and $d_{53}$ can be calculated on the basis of response 
proportion. The value of $y_{52}$ and $y_{53}$ respectively will be the maximum of proportion-of-responses and $\mu_{52}$ and $\mu_{53}$ of 0.9 of the respective risk factors. The negative or positive attribute as defined earlier will determine whether the factor would contribute to the risk $\left(\mathrm{A}_{5}\right)$ or otherwise.

Suppose if same assumptions are made for risk factor $\left(d_{52}\right)$ with $25 \%$ of the respondents strongly agree, $25 \%$ of the respondents agree, $40 \%$ of the respondents disagree and $10 \%$ of respondents strongly disagree then their values of $\mathrm{d}_{521}, \mathrm{~d}_{522}, \mathrm{~d}_{523}$ and $\mathrm{d}_{524}$ would be $0.25,0.25,0.4$ and 0.1 respectively. $\mu_{52}$ being 0.9 the value of $y_{52}$ will be the maximum of $\left(0.25^{*} 0.9\right),\left(0.25^{*} 0.9\right),(0.4 * 0.9)$ and $(0.1 * 0.9)$ which is 0.36 . Since the sum of SA and A is equal to $\mathrm{D}$ and $\mathrm{SD}$, this factor will not be taken into consideration for determination of risk $\left(\mathrm{A}_{5}\right)$.

In the second stage, an evaluation for each of the five risks identified in the acquisition stage will be determined by taking into consideration the values of risk factors as given in the Table 2:

- *Responses spread evenly throughout the given response-options, hence not considered

- $\quad * *$ Negative denotes that the responses relating to SA and/or $\mathrm{A}$ is greater than SD and/or D and hence will contribute towards risk. While positive denotes responses relating to $\mathrm{SD}$ and $\mathrm{D}$ that will not contribute to the risk. Y values with negative perspectives only will be considered for risk measurement

While measuring the values of the risk factor only negative perspectives of responses namely Strongly Agree and Agree will be considered for contributing towards risk. All these values will contribute towards the risk identified in the acquisition stage of ERP Adoption. In other words, the lowest value amongst the risk factors will determine the minimum amount of risk in the acquisition phase of ERP Adoption. In the above analysis various scenarios are explained to illustrate the mix of responses between the four options namely SA, A, SD and D in order to arrive at an understanding. This understanding can be validated in reality during the field survey.

\section{CONCLUSION}

The aim of this study is to provide a conceptual model using FPN for ERP failure prediction due to risks in the acquisition phase of ERP Adoption in the Small and Medium Enterprises. The acquisition phase is the second phase of ERP Adoption after the planning phase and is followed by three subsequent phases namely Implementation, Usage and Percolation and Extension. This conceptual model will be tested with live data by means of a survey to validate its applicability in real-life business. This risk prediction model using FPN can be further extended to all the other four phases of ERP adoption to arrive at a comprehensive risk evaluation and measurement model for ERP Adoption in SMEs. Moreover the possibility of developing a software tool is in progress as part of the ongoing project using the synergies of spreadsheet modeling with Visual Basic for Applications (VBA). This tool can embed features like colour-coding in red and green in order to highlight the degree of contributing or not contributing towards a particular risk in any of the phases of ERP adoption. The tool enabled with comprehensive risk evaluation and measurement model will be introduced to the SME industry associations. We believe that by training the industry associations on the understanding of the tool, it can be used as a reference for testing out the risk perceptions of ERP Adoption at various levels in different SME clusters across geographies.

\section{REFERENCES}

Amalnick, M.S., A.A.S. Ansarinejad and K. HatamiShirkouhi, 2010. A group decision making approach for evaluation of erp critical success factors using fuzzy AHP. Proceedings of the Fourth UKSim European Symposium on Computer Modeling and Simulation, Nov. 17-19, IEEE Xplore Press, Pisa, pp: 212-217. DOI: 10.1109/EMS.2010.42

Aman, D., G., Peter and B. Neil, 2007. Investigating factors affecting ERP selection in made-to-order SME sector. J. Manufacturing Technol. Manag., 19: 430-446.

Amin, A., M. Moalagh and A.Z. Ravasan, 2012. Identification and classification of ERP critical failure factors in Iranian industries. Inform. Syst., 227-237. DOI: 10.1016/j.is.2011.10.010

Ganesh, L. and A. Mehta, 2010. Critical failure factors in enterprise resource planning implementation at Indian SMEs. Asian J. Manage. Res., 1: 44-57.

Karsak, E.E. and C.O. Ozogul, 2009. An integrated decision making approach for ERP system selection. Exp. Syst. Applic., 36: 660-667. DOI: 10.1016/j.eswa.2007.09.016

Razmi, J., M.S. Sangari and R. Ghodsi, 2009. Developing a practical framework for ERP readiness assessment using fuzzy analytic network process. Adv. Eng. Software, 40: 1168-1178. DOI: 10.1016/j.advengsoft.2009.05.002 
Sen, C.G., H. Baraclı, S. Sen and H. Basligil, 2009. An integrated decision support system dealing with qualitative and quantitative objectives for enterprise software selection. Exp. Syst. Applic., 36: 52725283. DOI: 10.1016/j.eswa.2008.06.070

Somers, T.M. and K.G. Nelson, 2004. A taxonomy of players and activities across the ERP project life cycle. Inform. Manage., 41: 257-278. DOI: 10.1016/S0378-7206(03)00023-5

Vijayakumar, B. and P. Shrikant, 2009. A unified theory of critical success factors for ERP adoption by SMEs. Proceedings of the International Conference on Global Interdependence in Decision Sciences, (ICGIDS' 09), ASCI, Hyderabad, India.

Vijayakumar, B. and P. Shrikant, 2012. A comparative study on the conceptual and contextual perception about CSF for ERP adoption in the SMEs. Res. World J. Arts Sci. Commerce, 3: 38-46.
Vijayakumar, B., P. Dhanya and R. Ramakrishnan, 2012a. A conceptual model for erp failure prediction using fuzzy petri-nets for small and medium enterprises. Eur. J. Sci. Res., 87: 330-338.

Vijayakumar, B.S., V. Omkarprasad and P. Shrikant, 2012b. Prioritizing and ranking critical success factors for ERP adoption in SMEs. AIMS Int. J. Manage., 6: 23-40.

Wei, C.C., C. Chien and M.J. Wang, 2005. An AHPbased approach to ERP system selection. Int. J. Product. Econ., 96: 47-62. DOI: 10.1016/j.ijpe.2004.03.004

You, C.J., C.K.M. Lee, S.L. Chen and R.J. Jiao, 2012. A real option theoretic fuzzy evaluation model for enterprise resource planning investment. J. Eng. Technol. Manage., 29: 47-61. DOI: 10.1016/j.jengtecman.2011.09.005 